Mingyou Zhang and Md Salik Ahmed*

\title{
Sharp conditions of global existence for nonlinear Schrödinger equation with a harmonic potential
}

https://doi.org/10.1515/anona-2020-0031

Received January 18, 2019; accepted February 25, 2019.

Abstract: The Cauchy problem of nonlinear Schrödinger equation with a harmonic potential for describing the attractive Bose-Einstein condensate under the magnetic trap is considered. We give some sufficient conditions of global existence and finite time blow up of solutions by introducing a family of potential wells. Some different sharp conditions for global existence, and some invariant sets of solutions are also obtained here.

Keywords: nonlinear Schrödinger equation; harmonic potential; potential wells; global existence; invariant sets

\section{Introduction}

In the present paper we study the Cauchy problem of nonlinear Schrödinger equation with a harmonic potential

$$
\left\{\begin{array}{l}
i \varphi_{t}=-\Delta \varphi+|x|^{2} \varphi-|\varphi|^{p-1} \varphi, x \in \mathbb{R}^{n}, t>0 \\
\varphi(x, 0)=\varphi_{0}(x), x \in \mathbb{R}^{n}
\end{array}\right.
$$

where $p$ satisfies

$$
\text { (A) } 1+\frac{4}{n}<p<\frac{n+2}{n-2} \text { for } n \geq 3 ; 1+\frac{4}{n}<p<\infty \text { for } n=1,2 \text {. }
$$

The equation in (1.1) may model the Bose-Einstein condensate with attractive inter-particle interactions under the magnetic trap $[1,6,10,11]$.

Fujiwara [7] considered the above nonlinear Schrödinger equation with a general real-valued potential function $V(x)$ which later studied in [5]. When $\left|D^{\alpha} V(x)\right|$ is bounded for all $\alpha \geq 2$, the author of [7] gave the smoothness of Schrödinger kernel for potentials of quadratic growth. Further Yajima [20] showed that for super-quadratic potentials, the Schrödinger kernel is nowhere $C^{1}$. In addition, Oh [16] pointed out that the quadratic potentials are the highest order potentials for local well-posedness of the equation. Therefore $V(x)=|x|^{2}$ is the critical potential for the local existence of the Cauchy problem.

On the global existence and finite time blow up of solutions for problem (1.1), there have been some results. Firstly, Oh [16] and Cazenave [4] established the local existence of solutions of problem (1.1) in the energy space. Then for $1<p<1+\frac{4}{n}$, Zhang [22] proved the global existence of solutions for any initial data in energy space. For the case $p=1+\frac{4}{n}$, Zhang [21] gave a sharp condition of global existence of solution for problem (1.1). For the case $p>1+\frac{4}{n}$, Cazenave [4], Carles [2, 3] and Tsurumi and Wadati [18] showed that the solutions of problem (1.1) blows up in a finite time for some initial data, especially for a class of

Mingyou Zhang, College of Science, Northwest A\&F University, Yangling Shaanxi 712100, People's Republic of China, E-mail: zhangmingyouheu@163.com

*Corresponding Author: Md Salik Ahmed, Department of Mathematics and Physics, North South University, Dhaka-1229, Bangladesh, E-mail: salik.ahmed@northsouth.edu 
sufficiently large initial data; but the solutions of problem (1.1) globally exist for other sufficiently small initial data $[2,3,18]$.

Chen and Zhang [5] studied problem (1.1) and gave a sufficient condition of global existence of solutions in energy space. They proved that if $p$ satisfies (A), $\varphi_{0} \in H\left(\mathbb{R}^{n}\right)$ and satisfies $\left\|\varphi_{0}\right\|_{L^{2}} \leq h\left(\left\|\nabla \varphi_{0}\right\|_{L^{2}}^{2}+\left\|\varphi_{0}\right\|_{L^{2}}^{2}\right)$, then the solution $\varphi$ of problem (1.1) exists globally and satisfies

$$
\|\nabla \varphi\|_{L^{2}}^{2}+\||x| \varphi\|_{L^{2}}^{2} \leq \frac{2 n(p-1)}{n(p-1)-4} E\left(\varphi_{0}\right),
$$

where

$$
\begin{aligned}
H\left(\mathbb{R}^{n}\right) & =\left\{\varphi \in H ^ { 1 } ( \mathbb { R } ^ { n } ) \left|\|x \mid \varphi\|_{\left.L^{2}<\infty\right\},}<\right.\right. \\
h(\lambda) & =\left(\frac{n(p-1)-4}{4-(p-1)(n-2)}\right)^{\frac{n(p-1)-4}{2(n+2)-(n-2) p)}} \cdot\|Q\|_{2}^{\frac{2(p-1)}{(n+2)-(n-2) p}} \lambda^{-\frac{n(p-1)-4}{2(n+2)-(n-2) p)}}, \\
E(\varphi) & =\frac{1}{2}\|\nabla \varphi\|_{L^{2}}^{2}+\frac{1}{2}\|x \mid \varphi\|_{L^{2}}^{2}-\frac{1}{p+1}\|\varphi\|_{L^{p+1}}^{p+1},
\end{aligned}
$$

$Q$ is the ground state solution of equation

$$
-\Delta u+u=|u|^{p-1} u \text { in } \mathbb{R}^{n} .
$$

In addition, in [17] Shu and Zhang gave a sharp condition for global existence of solution for problem (1.1). Moreover, in [23] Zhang studied Cauchy problem of following nonlinear Schrödinger equation with harmonic potential

$$
i \varphi_{t}=-\frac{1}{2} \Delta \varphi+\hbar^{2}|x|^{2} \varphi-|\varphi|^{p-1} \varphi, x \in \mathbb{R}^{n}, t>0,
$$

obtained a sharp condition for global existence and finite time blow up of solutions and discussed the instability of standing wave. These works motivated us to study on this and related problems [24-32].

In this paper we study problem (1.1), where $p$ satisfies (A). By introducing two families of sets $W_{\delta}$ and $V_{\delta}$ like [12-14], we not only give some different sufficient conditions for global existence and finite time blow up of solutions which are completely different from that given in [2-5], [17], [18], [23] but also obtain a lot of different sharp conditions for global existence of solutions and some invariant sets of solutions for problem (1.1).

Throughout the present paper, the following notations are used for precise statements: $L^{p}\left(\mathbb{R}^{n}\right)(1 \leq p \leq \infty)$ denotes the usual space of all complex $L^{p}$-functions on $\mathbb{R}^{n}$ with norm $\|\varphi\|_{L^{p}\left(\mathbb{R}^{n}\right)}=\|\varphi\|_{p}$ and $\|\varphi\|_{L^{2}\left(\mathbb{R}^{n}\right)}=\|\varphi\|$. $H^{1}$ and $H$ denote $H^{1}\left(\mathbb{R}^{n}\right)$ and $H\left(\mathbb{R}^{n}\right)$ respectively. $H\left(\mathbb{R}^{n}\right)$ and $E(\varphi)$ are defined by (1.2) and (1.4) respectively.

Propostion 1.1. $[4,8,9,16]$ Assume that $1<p<\frac{n+2}{n-2}$ for $n \geq 3$ and $1<p<\infty$ for $n=1,2$, and $\varphi_{0} \in$ $H^{1}\left(\mathbb{R}^{n}\right)$. Then the problem (1.1) admits a unique solution $\varphi(t) \in C\left([0, T) ; H\left(\mathbb{R}^{n}\right)\right.$ ) for some $T \in[0, \infty)$ (Maximal existence time), and $\varphi(t)$ satisfies the following two conservation laws:

$$
\|\varphi(t)\|=\left\|\varphi_{0}\right\|
$$

and

$$
E(\varphi(t))=E\left(\varphi_{0}\right)
$$

for all $t \in[0, T)$. Furthermore, we have the following alternative: $T=\infty$ or $T<\infty$ and

$$
\lim _{t \rightarrow T}\|\varphi\|_{H\left(\mathbb{R}^{n}\right)}=\infty .
$$

Propostion 1.2. [4] Let $\varphi(t)$ be a solution of problem (1.1) with $\varphi_{0} \in H$, and

$$
F(t)=\int|x|^{2}|\varphi|^{2} \mathrm{~d} x
$$

Then $\varphi(t) \in H$ for $0 \leq t<T$ and

$$
F^{\prime \prime}(t)=8 \int\left(|\nabla \varphi|^{2}-|x|^{2}|\varphi|^{2}-\frac{n(p-1)}{2(p+1)}|\varphi|^{p+1}\right) \mathrm{d} x, 0 \leq t<T
$$


and

$$
\|\varphi\|^{2} \leq \frac{2}{n}\|\nabla \varphi\| F^{\frac{1}{2}}(t), 0 \leq t<T .
$$

Propostion 1.3. [19] Let $1<p<\frac{n+2}{n-2}$ for $n \geq 3$ and $1<p<\infty$ for $n=1,2$. Then the best constant $C_{\star}>0$ of the Gagliardo-Nirenberg's inequality,

$$
\|f\|_{L^{p+1}}^{p+1} \leq C_{\star}\|f\|_{L^{2}}^{p+1-\frac{n(p-1)}{2}}\|\nabla f\|_{L^{2}}^{\frac{n(p-1)}{2}},
$$

is given by

$$
C_{\star}=\frac{2(p+1)}{n(p-1)}\left(\frac{4-(p-1)(n-2)}{n(p-1)}\right)^{\frac{n(p-1)-4}{4}}\|Q\|_{L^{2}}^{-(p-1)} .
$$

\section{Preliminaries}

In this section we shall give some necessaries lemmas and by using them we introduce two families $W_{\delta}$ and $V_{\delta}$. For problem (1.1) with $\left\|\varphi_{0}\right\| \neq 0$ we define

$$
\begin{aligned}
\|\varphi\|_{H}^{2}=\|\varphi\|_{H^{1}}^{2} & +\||x| \varphi\|_{L^{2}}^{2}=\|\nabla \varphi\|^{2}+\|\varphi\|^{2}+\||x| \varphi\|^{2}, \\
\widetilde{H} & =\left\{\varphi \in H \mid\|\varphi\|=\left\|\varphi_{0}\right\|\right\}, \\
J(\varphi) & =\frac{1}{2}\|\nabla \varphi\|^{2}-\frac{1}{p+1}\|\varphi\|_{p+1}^{p+1}, \\
I_{\delta}(\varphi) & =\delta\|\nabla \varphi\|^{2}-\|\varphi\|_{p+1}^{p+1}, \delta>0 .
\end{aligned}
$$

From (1.7) we can obtain the following lemma.

Lemma 2.1. Let $p$ satisfy $(A), \varphi \in \widetilde{H}$. Then $\|\varphi\|_{p+1} \neq 0$ and $\|\nabla \varphi\| \neq 0$.

Next we discuss the relations between $\|\nabla \varphi\|$ and the sign of $I_{\delta}(\varphi)$, which are crucial for obtaining the main results in this paper.

Lemma 2.2. Let $p$ satisfy (A). Assume that $\varphi \in \widetilde{H}$ and

$$
r(\delta)=\left(\frac{\delta}{C_{\star} M_{0}}\right)^{\frac{1}{q}}, M_{0}=\left\|\varphi_{0}\right\|^{p+1-\frac{n(p-1)}{2}}, q=\frac{n(p-1)}{2}-2 .
$$

(i) If $\|\nabla \varphi\|<r(\delta)$, then $I_{\delta}(\varphi)>0$.

(ii) If $I_{\delta}(\varphi)<0$, then $\|\nabla \varphi\|>r(\delta)$.

(ii) If $I_{\delta}(\varphi)=0$, then $\|\nabla \varphi\| \geq r(\delta)$.

Proof.

(i) Since $\varphi \in \widetilde{H}$ implies $\|\nabla \varphi\| \neq 0$, from $\|\nabla \varphi\|<r(\delta)$ we get

$$
\|\varphi\|_{p+1}^{p+1} \leq C_{\star}\|\varphi\|^{p+1-\frac{n(p-1)}{2}}\|\nabla \varphi\|^{\frac{n(p-1)}{2}}=C_{\star} M_{0}\|\nabla \varphi\|^{q}\|\nabla \varphi\|^{2}<\delta\|\nabla \varphi\|^{2}
$$

which gives $I_{\delta}(\varphi)>0$.

(ii) From $I_{\delta}(\varphi)<0$ we get

$$
\delta\|\nabla \varphi\|^{2}<\|\varphi\|_{p+1}^{p+1} \leq C_{\star} M_{0}\|\nabla \varphi\|^{q}\|\nabla \varphi\|^{2},
$$

which gives $\|\nabla \varphi\|>r(\delta)$. 
(iii) From $I_{\delta}(\varphi)=0$ we get

$$
\delta\|\nabla \varphi\|^{2}=\|\varphi\|_{p+1}^{p+1} \leq C_{\star} M_{0}\|\nabla \varphi\|^{q}\|\nabla \varphi\|^{2},
$$

which together with $\|\nabla \varphi\| \neq 0$ gives $\|\nabla \varphi\| \geq r(\delta)$.

As it is well known that in space $H^{1}\left(\mathbb{R}^{n}\right)$, Poincare inequality does not hold, so that one can not use the important fact that $\|\nabla u\|$ is equivalent to $\|u\|_{H^{1}}$. In order to overcome this difficulty, we introduce the space $\widetilde{H}\left(\mathbb{R}^{n}\right)$, so that by (1.5) and (1.7) the norms $\|\nabla \varphi\|$ and $\|\varphi\|_{H^{1}}$ are equivalent in some sense again.

Definition 2.3. For problem (1.1) with $\left\|\varphi_{0}\right\| \neq 0$ we define

$$
d(\delta)=\inf _{\varphi \in \mathcal{N}_{\delta}} J(\varphi), \mathcal{N}_{\delta}=\left\{\varphi \in \widetilde{H} \mid I_{\delta}(\varphi)=0\right\}, \delta>0 .
$$

In the following Lemma 2.4 we estimate the value of $d(\delta)$ and give its expression by $d(1)$, which palys an important role in the proof of the main results of this paper.

Lemma 2.4. Let $p$ satisfy $(A)$. Then

(i) $d(\delta) \geq a(\delta) r^{2}(\delta)$ for $a(\delta)=\frac{1}{2}-\frac{\delta}{p+1}, 0<\delta<\frac{p+1}{2}$;

(ii) $d(\delta)=\delta^{\frac{4}{(p-1)-4}} \frac{p+1-2 \delta}{p-1} d(1), 0<\delta<\frac{p+1}{2}$.

$\operatorname{Pr}(\dot{a})$ f. For any $\varphi \in \mathcal{N}_{\delta}, 0<\delta<\frac{p+1}{2}$ we have $\|\nabla \varphi\| \geq r(\delta)$ and

$$
\begin{aligned}
J(\varphi) & =\frac{1}{2}\|\nabla \varphi\|^{2}-\frac{1}{p+1}\|\varphi\|_{p+1}^{p+1} \\
& =\left(\frac{1}{2}-\frac{\delta}{p+1}\right)\|\nabla \varphi\|^{2}+\frac{1}{p+1} I_{\delta}(\varphi) \\
& =a(\delta)\|\nabla \varphi\|^{2} \geq a(\delta) r^{2}(\delta),
\end{aligned}
$$

which gives $d(\delta) \geq a(\delta) r^{2}(\delta)$ for $0<\delta<\frac{p+1}{2}$.

(ii) (a) From the definition of $d(1)$ it follows that for any $\varepsilon>0$ there exists a $\varphi \in \mathcal{N}_{1}$ such that

$$
d(1) \leq J(\varphi)<d(1)+\varepsilon .
$$

For $\delta>0$, define $\lambda=\lambda(\delta)$ by

$$
\delta\left\|\nabla \varphi^{\lambda}\right\|^{2}=\left\|\varphi^{\lambda}\right\|_{p+1}^{p+1}, \varphi^{\lambda}(x)=\lambda^{\frac{n}{2}} \varphi(\lambda x)
$$

Then

$$
\delta\|\nabla \varphi\|^{2}=\lambda^{\frac{n(p-1)-4}{2}}\|\varphi\|_{p+1}^{p+1} .
$$

Hence for each $\delta>0$ there exists a unique

$$
\lambda(\delta)=\left(\frac{\delta a(\varphi)}{b(\varphi)}\right)^{\frac{2}{n(p-1)-4}},
$$

where

$$
a(\varphi)=\|\nabla \varphi\|^{2}, b(\varphi)=\|\varphi\|_{p+1}^{p+1} .
$$

Since $\varphi \in \mathcal{N}_{1}$ implies $a(\varphi)=b(\varphi)$ we get

$$
\lambda(\delta)=\delta^{\frac{2}{(p-1)-4}} .
$$

Note that $\left\|\varphi^{\lambda}\right\|=\|\varphi\|=\left\|\varphi_{0}\right\|, \forall \lambda>0$, we have $\varphi \lambda(\delta) \in \mathcal{N}_{\delta}$. From the definition of $d(\delta)$ we get

$$
\begin{aligned}
d(\delta) & \leq J\left(\varphi^{\lambda}\right)=\frac{1}{2} \lambda^{2} a(\varphi)-\frac{1}{p+1} \lambda^{\frac{n(p-1)}{2}} b(\varphi) \\
& =\frac{1}{2} \delta^{\frac{4}{n(p-1)-4}} a(\varphi)-\frac{1}{p+1} \delta^{\frac{n(p-1)}{n(p-1)-4}} b(\varphi) \\
& =\delta^{\frac{4}{n(p-1)-4}}\left(\frac{1}{2}-\frac{\delta}{p+1}\right) a(\varphi) .
\end{aligned}
$$


From (2.1) and

$$
J(\varphi)=\frac{1}{2} a(\varphi)-\frac{1}{p+1} b(\varphi)=\frac{p-1}{2(p+1)} a(\varphi)
$$

it follows that

$$
\begin{aligned}
d(\delta) & \leq \delta^{\frac{4}{n(p-1)-4}}\left(\frac{1}{2}-\frac{\delta}{p+1}\right) \frac{2(p+1)}{p-1} J(\varphi) \\
& <\delta^{\frac{4}{n(p-1)-4}}\left(\frac{1}{2}-\frac{\delta}{p+1}\right) \frac{2(p+1)}{p-1}(d(1)+\varepsilon), 0<\delta<\frac{p+1}{2} .
\end{aligned}
$$

From the arbitrariness of $\varepsilon$ we obtain

$$
d(\delta) \leq \delta^{\frac{4}{n(p-1)-4}} \frac{p+1-2 \delta}{p-1} d(1), 0<\delta<\frac{p+1}{2} .
$$

(b) Let $\delta>0$. From the definition of $d(\delta)$ it follows that for any $\varepsilon>0$ there exists a $\varphi \in \mathcal{N}_{\delta}$ such that

$$
d(\delta) \leq J(\varphi)<d(\delta)+\varepsilon
$$

Define $\lambda=\lambda(\delta)$ by

$$
\left\|\nabla \varphi^{\lambda}\right\|^{2}=\left\|\varphi^{\lambda}\right\|_{p+1}^{p+1}
$$

Then

$$
\begin{gathered}
\|\nabla \varphi\|^{2}=\lambda^{\frac{n(p-1)-4}{2}}\|\varphi\|_{p+1}^{p+1} . \\
\lambda=\left(\frac{a(\varphi)}{b(\varphi)}\right)^{\frac{2}{n(p-1)-4}} .
\end{gathered}
$$

Since $\varphi \in \mathcal{N}_{\delta}$ implies that $\delta a(\varphi)=b(\varphi)$ we get

$$
\lambda(\delta)=\left(\frac{1}{\delta}\right)^{\frac{2}{n(p-1)-4}}
$$

From $\varphi^{\lambda} \in \mathcal{N}_{1}$ and the definition of $d(1)$ we have

$$
\begin{aligned}
d(1) & \leq J\left(\varphi^{\lambda}\right)=\frac{1}{2} \lambda^{2} a(\varphi)-\frac{1}{p+1} \lambda^{\frac{n(p-1)}{2}} b(\varphi) \\
& =\frac{1}{2}\left(\frac{1}{\delta}\right)^{\frac{4}{n(p-1)-4}} a(\varphi)-\frac{1}{p+1}\left(\frac{1}{\delta}\right)^{\frac{n(p-1)}{n(p-1)-4}} b(\varphi) \\
& =\left(\frac{1}{\delta}\right)^{\frac{4}{n(p-1)-4}}\left(\frac{1}{2} a(\varphi)-\frac{1}{p+1} \frac{1}{\delta} b(\varphi)\right) \\
& =\left(\frac{1}{\delta}\right)^{\frac{4}{n(p-1)-4}} \frac{p-1}{2(p+1)} a(\varphi) .
\end{aligned}
$$

From (2.3) and

$$
J(\varphi)=\frac{1}{2} a(\varphi)-\frac{1}{p+1} b(\varphi)=\left(\frac{1}{2}-\frac{\delta}{p+1}\right) a(\varphi)
$$

it follows that

$$
\begin{aligned}
d(\delta) & \leq\left(\frac{1}{\delta}\right)^{\frac{4}{n(p-1)-4}} \frac{p-1}{2(p+1)}\left(\frac{1}{2}-\frac{\delta}{p+1}\right)^{-1} J(\varphi) \\
& <\left(\frac{1}{\delta}\right)^{\frac{4}{n(p-1)-4}} \frac{p-1}{2(p+1)}\left(\frac{1}{2}-\frac{\delta}{p+1}\right)^{-1}(d(\delta)+\varepsilon), 0<\delta<\frac{p+1}{2}
\end{aligned}
$$

and

$$
d(\delta)+\varepsilon>\delta^{\frac{4}{n(p-1)-4}}\left(\frac{1}{2}-\frac{\delta}{p+1}\right) \frac{2(p+1)}{p-1} d(1), 0<\delta<\frac{p+1}{2}
$$


From (2.4) and the arbitrariness of $\varepsilon$ we get

$$
d(\delta) \geq \delta^{\frac{4}{n(p-1)-4}} \frac{p+1-2 \delta}{p-1} d(1), 0<\delta<\frac{p+1}{2} .
$$

From(2.2) and (2.5) we obtain (ii) of this lemma.

Corollary 2.5. Let $p$ satisfy (A). Then

(i) $\lim _{\delta \rightarrow 0} d(\delta)=0, \lim _{\delta \rightarrow \frac{p+1}{2}} d(\delta)=0$;

(ii) $d(\delta)$ is continuous on $0<\delta<\frac{p+1}{2}$;

(iii) $d(\delta)$ is increasing on $0<\delta \leq a$, decreasing on $a \leq \delta<\frac{p+1}{2}$ and takes the maximum $d(a)$ at $\delta=a=\frac{2(p+1)}{n(p-1)}$.

Proof. Conclusions (i) and (ii) follow from (ii) in Lemma 2.4 immediately.

Conclusion (iii) follows from (ii) in Lemma 2.4 and

$$
d^{\prime}(\delta)=A(a-\delta) \delta^{\alpha}, A=\frac{2 n}{n(p-1)-4} d(1), \alpha=\frac{\delta-n(p-1)}{n(p-1)-4} .
$$

Definition 2.6. For problem (1.1) with $\left\|\varphi_{0}\right\| \neq 0$ we define

$$
\begin{gathered}
I(\varphi)=I_{a}(\varphi), d=d(a), a=\frac{2(p+1)}{n(p-1)}, \\
W=\{\varphi \in \widetilde{H} \mid I(\varphi)>0, J(\varphi)<d\}, \\
V=\{\varphi \in \widetilde{H} \mid I(\varphi)<0, J(\varphi)<d\}, \\
W_{\delta}=\left\{\varphi \in \widetilde{H} \mid I_{\delta}(\varphi)>0, J(\varphi)<d(\delta)\right\}, 0<\delta<\frac{p+1}{2}, \\
V_{\delta}=\left\{\varphi \in \widetilde{H} \mid I_{\delta}(\varphi)<0, J(\varphi)<d(\delta)\right\}, 0<\delta<\frac{p+1}{2} .
\end{gathered}
$$

Remark 2.7. Lemma 2.4 shows that the depth $d$ of the potential well $W$ defined by Definition 2.6 depends on $\left\|\varphi_{0}\right\|$ and $d \rightarrow+\infty$ as $\left\|\varphi_{0}\right\| \rightarrow 0$. This property of $d$ is completely different from the depth of other known potential wells defined for other nonlinear evolution equations. In addition, $W$ or any $W_{\delta}$ do not include $\varphi=0$.

\section{Invariant sets and vacuum isolating of solutions}

In this section we discuss the invariant sets and vacuum isolating of solutions for problem (1.1). First we consider the case $0<E\left(\varphi_{0}\right)<d$, and the case $E\left(\varphi_{0}\right)=d$ will be considered later.

Theorem 3.1. Let $p$ satisfy $(A), \varphi_{0} \in H$. Assume that $0<e<d, \delta_{1}<\delta_{2}$ are two roots of equation $d(\delta)=e$. Then

(i) All solutions of problem (1.1) with $E\left(\varphi_{0}\right)=$ e belong to $W_{\delta}$ for $\delta \in\left[\delta_{1}, \delta_{2}\right]$, provided $I\left(\varphi_{0}\right)>0$.

(ii) All solutions of problem (1.1) with $E\left(\varphi_{0}\right)=e$ belong to $V_{\delta}$ for $\delta \in\left[\delta_{1}, \delta_{2}\right]$, provided $I\left(\varphi_{0}\right)<0$.

Proof.

(i) Let $\varphi(t) \in C([0, T) ; \tilde{H})$ be any solution of problem (1.1) with $E\left(\varphi_{0}\right)=e$ and $I\left(\varphi_{0}\right)>0$, $T$ be the existence time of $\varphi(t)$. Firstly we prove $\varphi_{0} \in W_{\delta}$ for $\delta \in\left[\delta_{1}, \delta_{2}\right]$. From

$$
\frac{1}{2}\left\||x| \varphi_{0}\right\|^{2}+J\left(\varphi_{0}\right)=E\left(\varphi_{0}\right)=e \leq d(\delta), \delta \in\left[\delta_{1}, \delta_{2}\right]
$$


we get $J\left(\varphi_{0}\right)<d(\delta)$ for $\delta \in\left[\delta_{1}, \delta_{2}\right]$. On the other hand, $I\left(\varphi_{0}\right)>0$ implies $\left\|\varphi_{0}\right\| \neq 0$. Hence from (3.1) we can get $I_{\delta}\left(\varphi_{0}\right)>0$ for $\delta \in\left[\delta_{1}, \delta_{2}\right]$. Otherwise there exists a $\bar{\delta} \in\left[\delta_{1}, \delta_{2}\right]$ such that $I_{\bar{\delta}}\left(\varphi_{0}\right)=0$ which together with $\left\|\varphi_{0}\right\| \neq 0$ gives $J\left(\varphi_{0}\right) \geq d(\bar{\delta})$. This contradicts (3.1). Next we prove that $\varphi(t) \in W_{\delta}$ for $\delta \in\left[\delta_{1}, \delta_{2}\right]$, $t \in(0, T)$. Arguing by contradiction, we suppose that there exists a $t_{0} \in(0, T)$ such that $\varphi\left(t_{0}\right) \in \partial W_{\delta}$ for some $\delta \in\left[\delta_{1}, \delta_{2}\right]$, i.e. $I_{\delta}\left(\varphi\left(t_{0}\right)\right)=0$ or $J\left(\varphi\left(t_{0}\right)\right)=d(\delta)$. From (1.6) we get

$$
\frac{1}{2}\||x| \varphi\|^{2}+J(\varphi)=E\left(\varphi_{0}\right) \leq d(\delta), \delta \in\left[\delta_{1}, \delta_{2}\right], t \in(0, T) .
$$

Hence $J\left(\varphi\left(t_{0}\right)\right)=d(\delta)$ is impossible. If $I_{\delta}\left(\varphi\left(t_{0}\right)\right)=0$, then by $\left\|\varphi\left(t_{0}\right)\right\|=\left\|\varphi_{0}\right\| \neq 0$ we get $J\left(\varphi\left(t_{0}\right)\right) \geq d(\delta)$ which contradicts (3.2).

(ii) Let $\varphi(t) \in C([0, T) ; \tilde{H})$ be any solution of problem (1.1) with $E\left(\varphi_{0}\right)=e, I\left(\varphi_{0}\right)<0, T$ be the existence time of $\varphi(t)$. From $I\left(\varphi_{0}\right)<0$ and (3.1) we can get $\varphi_{0} \in V_{\delta}$ for $\delta \in\left[\delta_{1}, \delta_{2}\right]$. The remainder of this proof is similar to that in part (i).

From (3.2) it follows that if $0<E\left(\varphi_{0}\right)=e<d, \delta_{1}<\delta_{2}$ are two roots of equation $d(\delta)=e$, then for any $\delta \in\left[\delta_{1}, \delta_{2}\right], \varphi \in \mathcal{N}_{\delta}$ is impossible. Therefore for the set of all solutions of problem (1.1) with $0<E\left(\varphi_{0}\right)=e<d$ there exists a vacuum region

$$
U_{e}=\mathcal{N}_{\delta_{1} \delta_{2}}=\bigcup_{\delta_{1} \leq \delta \leq \delta_{2}} \mathcal{N}_{\delta}=\left\{\varphi \in \widetilde{H} \mid I_{\delta}(\varphi)=0, \delta_{1} \leq \delta \leq \delta_{2}\right\}
$$

such that $\varphi(t) \notin U_{e}$ for any solution $\varphi(t)$ of problem (1.1) with $0<E\left(\varphi_{0}\right)=e<d$.

Now we consider the invariant sets of solutions of problem (1.1) with $E\left(\varphi_{0}\right)=d$.

Theorem 3.2. Let $p$ satisfy $(A), \varphi_{0} \in H$. Then

(i) All solutions of problem (1.1) with $E\left(\varphi_{0}\right)=d$ belong to $W$, provided $I\left(\varphi_{0}\right)>0$.

(ii) All solutions of problem (1.1) with $E\left(\varphi_{0}\right)=d$ belong to $V$, provided $I\left(\varphi_{0}\right)<0$.

$\operatorname{Pr}(\dot{\omega})$ f. Let $\varphi(t) \in C([0, T) ; \tilde{H})$ be any solution of problem (1.1) with $E\left(\varphi_{0}\right)=d$ and $I\left(\varphi_{0}\right)>0, T$ be the existence time of $\varphi(t)$. Firstly $I\left(\varphi_{0}\right)>0$ gives $\left\|\varphi_{0}\right\| \neq 0$ and $\left\||x| \varphi_{0}\right\| \neq 0$. Hence from

$$
J\left(\varphi_{0}\right)=E\left(\varphi_{0}\right)-\frac{1}{2}\left\||x| \varphi_{0}\right\|^{2}=d-\frac{1}{2}\left\||x| \varphi_{0}\right\|^{2}<d
$$

we get $\varphi_{0} \in W$. Next we prove that $\varphi(t) \in W$ for $t \in(0, T)$. Arguing by contradiction, we assume that there exists a $t_{0} \in(0, T)$ such that $\varphi\left(t_{0}\right) \in \partial W$, i.e. $I\left(\varphi\left(t_{0}\right)\right)=0$ or $J\left(\varphi\left(t_{0}\right)\right)=d$. From $\left\|\varphi\left(t_{0}\right)\right\|=\left\|\varphi_{0}\right\| \neq 0$ we get $\left\||x| \varphi\left(t_{0}\right)\right\| \neq 0$. Hence from

$$
J\left(\varphi\left(t_{0}\right)\right)=E\left(\varphi_{0}\right)-\frac{1}{2}\left\||x| \varphi\left(t_{0}\right)\right\|^{2}=d-\frac{1}{2}\left\||x| \varphi\left(t_{0}\right)\right\|^{2}<d
$$

we see that $J\left(\varphi\left(t_{0}\right)\right)=d$ is impossible. If $I\left(\varphi\left(t_{0}\right)\right)=0$, then again we have $J\left(\varphi\left(t_{0}\right)\right) \geq d$ which contradicts $J\left(\varphi\left(t_{0}\right)\right)<d$.

(ii) The proof is similar to that of part (i) of this lemma.

Next we discuss the invariant sets of solutions for problem (1.1) with $E\left(\varphi_{0}\right) \leq 0$.

Theorem 3.3. Let $p$ satisfy $(A), \varphi_{0} \in H$. Assume that $E\left(\varphi_{0}\right)<0$ or $E\left(\varphi_{0}\right)=0,\left\|\varphi_{0}\right\| \neq 0$. Then all solutions of problem (1.1) belong to $V_{\delta}$ for $\delta \in\left(0, \frac{p+1}{2}\right)$.

Proof. Let $\varphi(t)$ be any solution of problem (1.1) with $E\left(\varphi_{0}\right)<0$ or $E\left(\varphi_{0}\right)=0,\left\|\varphi_{0}\right\| \neq 0$. Since $E\left(\varphi_{0}\right)<0$ implies $\left\|\varphi_{0}\right\| \neq 0$. Hence for two cases we always have $\|\varphi(t)\|=\left\|\varphi_{0}\right\| \neq 0$ and $\|\nabla \varphi(t)\| \neq 0$ for $0 \leq t<T$. Thus from

$$
\begin{array}{r}
\left(\frac{1}{2}-\frac{\delta}{p+1}\right)\|\nabla \varphi\|^{2}+\frac{1}{p+1} I_{\delta}(\varphi)=J(\varphi)=E\left(\varphi_{0}\right)-\frac{1}{2}\||x| \varphi\|^{2}, \\
0<\delta<\frac{p+1}{2}, 0 \leq t<T,
\end{array}
$$


we can get $I_{\delta}(\varphi)<0$ and $J(\varphi)<0<d(\delta)$ for $\delta \in\left(0, \frac{p+1}{2}\right), t \in(0, T)$, which gives $\varphi(t) \in V_{\delta}$ for $\delta \in\left(0, \frac{p+1}{2}\right)$, $t \in[0, T)$.

\section{4 global existence and finite time blow up of solutions}

In this section we shall prove the global existence and finite time blow up of solutions and give some sharp conditions for global existence and finite time blow up of solutions for problem (1.1) which are completely different from those given in [2-5], [17, 18, 23].

Theorem 4.1. Let $p$ satisfy $(A), \varphi_{0} \in H$. Assume that $\left\|\varphi_{0}\right\|=0$ or $E\left(\varphi_{0}\right) \leq d, I\left(\varphi_{0}\right)>0$. Then problem (1.1) admits a unique global solution $\varphi(t) \in C([0, \infty) ; \widetilde{H})$ such that

(i) $\|\varphi(t)\|=\left\|\varphi_{0}\right\|=0$ for $0 \leq t<\infty$ if $\left\|\varphi_{0}\right\|=0$.

or

(ii) $\varphi(t) \in W$ for $0 \leq t<\infty$ if $E\left(\varphi_{0}\right) \leq d, I\left(\varphi_{0}\right)>0$.

Proof. Firstly from Proposition 1.1 it follows that problem (1.1) admits a unique local solution $\varphi(t) \in$ $C([0, T) ; H)$ satisfying (1.5), (1.6). Next we prove $T=+\infty$.

(i) If $\left\|\varphi_{0}\right\|=0$, then by (1.5) we have $\|\varphi(t)\|=\left\|\varphi_{0}\right\|=0,0 \leq t<T$, which gives $\||x| \varphi(t)\|=0$ and $\|\nabla \varphi(t)\|=0$, i.e. $\|\varphi(t)\|_{H}=0$ for $0 \leq t<T$. Hence by Proposition 1.1 we get $T=+\infty$.

(ii) If $E\left(\varphi_{0}\right) \leq d, I\left(\varphi_{0}\right)>0$, then

$$
E\left(\varphi_{0}\right)=\frac{1}{2}\left\||x| \varphi_{0}\right\|^{2}+\frac{n(p-1)-4}{2 n(p-1)}\left\|\nabla \varphi_{0}\right\|^{2}+\frac{1}{p+1} I\left(\varphi_{0}\right)>0 .
$$

Consequently, from Theorem 3.1 and Theorem 3.2 we have $\varphi(t) \in W$ for $0 \leq t<T$. Thus, from

$$
\begin{aligned}
& \frac{1}{2}\||x| \varphi\|^{2}+\frac{n(p-1)-4}{2 n(p-1)}\|\nabla \varphi\|^{2}+\frac{1}{p+1} I(\varphi) \\
= & \frac{1}{2}\||x| \varphi\|^{2}+J(\varphi)=E\left(\varphi_{0}\right), 0 \leq t<T,
\end{aligned}
$$

we get

$$
\||x| \varphi\|^{2}+\|\nabla \varphi\|^{2} \leq \frac{2 n(p-1)}{n(p-1)-4} E\left(\varphi_{0}\right), 0 \leq t<T
$$

and

$$
\||x| \varphi\|^{2}+\|\nabla \varphi\|^{2}+\|\varphi\|^{2} \leq \frac{2 n(p-1)}{n(p-1)-4} E\left(\varphi_{0}\right)+\left\|\varphi_{0}\right\|^{2}, 0 \leq t<T,
$$

which gives $T=+\infty$.

Since $I\left(\varphi_{0}\right)>0$ gives $E\left(\varphi_{0}\right)>0$, the following corollary is the improvement of Theorem 4.1.

Corollary 4.2. If in Theorem 4.1 the assumption " $E\left(\varphi_{0}\right) \leq d, I\left(\varphi_{0}\right)>0$ " is replaced by " $0<E\left(\varphi_{0}\right)<d$, $I_{\delta_{2}}\left(\varphi_{0}\right)>0$ ", where $\delta_{1}<\delta_{2}$ are two roots of equation $d(\delta)=E\left(\varphi_{0}\right)$, then problem (1.1) admits a unique global solution $\varphi(t) \in C([0, \infty) ; \widetilde{H})$ and $\varphi(t) \in W_{\delta}$ for $\delta \in\left[\delta_{1}, \delta_{2}\right], 0 \leq t<\infty$.

Proof. From Theorem 4.1 and Theorem 3.1 it follows that it is enough to prove $I\left(\varphi_{0}\right)>0$. In fact, if it is false, then there exists a $\bar{\delta} \in\left[a, \delta_{2}\right]$ such that $I_{\bar{\delta}}\left(\varphi_{0}\right)=0$ and $J\left(\varphi_{0}\right) \geq d(\bar{\delta})$, which contradicts (3.1).

Corollary 4.3. Let $p$ satisfy (A), $\varphi_{0} \in H, a<\delta_{0}<\frac{p+1}{2}$. Assume that $E\left(\varphi_{0}\right) \leq d\left(\delta_{0}\right)$ and $I_{\delta_{0}}\left(\varphi_{0}\right)>0$. Then problem (1.1) admits a unique global solution $\varphi(t) \in C([0, \infty) ; \widetilde{H})$ and $\varphi(t) \in W_{\delta_{0}}$ for $0 \leq t<\infty$. 
Proof. First we have $E\left(\varphi_{0}\right) \leq d\left(\delta_{0}\right)<d(a)=d$ and

$$
E\left(\varphi_{0}\right)=\frac{1}{2}\left\||x| \varphi_{0}\right\|^{2}+\left(\frac{1}{2}-\frac{\delta_{0}}{p+1}\right)\left\|\nabla \varphi_{0}\right\|^{2}+\frac{1}{p+1} I_{\delta_{0}}\left(\varphi_{0}\right)>0 .
$$

If $\delta_{1}<\delta_{2}$ are two roots of equation $d(\delta)=E\left(\varphi_{0}\right)$, then we have $\delta_{2} \geq \delta_{0}$. Hence $I_{\delta_{0}}\left(\varphi_{0}\right)>0$ gives $I_{\delta_{2}}\left(\varphi_{0}\right)>0$. So by Corollary 4.2, problem (1.1) admits a unique global solution $\varphi(t) \in C([0, \infty) ; \widetilde{H})$ and $\varphi(t) \in W_{\delta}$ for $\delta \in\left[\delta_{1}, \delta_{2}\right]$. Since $\delta_{0} \in\left(a, \delta_{2}\right] \subset\left[\delta_{1}, \delta_{2}\right]$ we obtain $\varphi(t) \in W_{\delta_{0}}$ for $0 \leq t<\infty$.

In the following theorem we give two results on global existence of solutions for problem (1.1) regarding $\left\|\nabla \varphi_{0}\right\|$.

Theorem 4.4. If in Corollary 4.2 the assumption " $I_{\delta_{2}}\left(\varphi_{0}\right)>0$ " is replaced by " $\left\|\nabla \varphi_{0}\right\|<r\left(\delta_{2}\right)$ ", then problem (1.1) admits a unique global solution $\varphi(t) \in C([0, \infty) ; \widetilde{H})$ satisfying

$$
\|\nabla \varphi\|^{2} \leq \frac{E\left(\varphi_{0}\right)}{a\left(\delta_{1}\right)},\||x| \varphi\|^{2} \leq 2 E\left(\varphi_{0}\right), 0 \leq t<\infty,
$$

where $r(\delta)$ and $a(\delta)$ are defined in Lemma 2.2 and Lemma 2.4 respectively.

Proof. Firstly $E\left(\varphi_{0}\right)>0$ gives $\left\|\varphi_{0}\right\| \neq 0$ and (by Lemma 2.1) $\left\|\nabla \varphi_{0}\right\| \neq 0$. Furthermore from $0<\left\|\nabla \varphi_{0}\right\|<r\left(\delta_{2}\right)$ we get $I_{\delta_{2}}\left(\varphi_{0}\right)>0$. Hence from Corollary 4.2 it follows that problem (1.1) admits a unique global solution $\varphi(t) \in C([0, \infty) ; \widetilde{H})$ and $\varphi(t) \in W_{\delta}$ for $\delta \in\left(\delta_{1}, \delta_{2}\right), 0 \leq t<\infty$. Finally in

$$
\frac{1}{2}\||x| \varphi\|^{2}+a(\delta)\|\nabla \varphi\|^{2}+\frac{1}{p+1} I_{\delta}(\varphi)=E\left(\varphi_{0}\right)
$$

letting $\delta=\delta_{1}$ we obtain (4.1).

Theorem 4.5. Let $p$ satisfy $(A), \varphi_{0} \in H$. Assume that $E\left(\varphi_{0}\right)=d,\left\|\nabla \varphi_{0}\right\|<r(a)$. Then problem (1.1) admits a unique global solution $\varphi(t) \in C([0, \infty) ; \widetilde{H})$ satisfying

$$
\|\nabla \varphi\|^{2} \leq \frac{2 n(p-1)}{n(p-1)-4} d,\||x| \varphi\|^{2} \leq 2 d, 0 \leq t<\infty .
$$

Proof. Firstly by $E\left(\varphi_{0}\right)=d>0$ we have $\left\|\varphi_{0}\right\| \neq 0$. Hence by Lemma 2.2 we get $I\left(\varphi_{0}\right)>0$. From Theorem 4.1 it follows that problem (1.1) admits a unique global solution $\varphi(t) \in C([0, \infty) ; \widetilde{H})$ and $\varphi(t) \in W$ for $0 \leq t<\infty$. Inequality (4.2) follows from

$$
\frac{1}{2}\||x| \varphi\|^{2}+\frac{n(p-1)-4}{2 n(p-1)}\|\nabla \varphi\|^{2}+\frac{1}{p+1} I(\varphi)=E\left(\varphi_{0}\right)=d, 0 \leq t<\infty .
$$

Next we discuss the finite time blow of solution for problem (1.1).

Theorem 4.6. Let $p$ satisfy $(A), \varphi_{0} \in H$. Assume that $E\left(\varphi_{0}\right)<d$ and $I\left(\varphi_{0}\right)<0$. Then the solution of problem (1.1) blows up in finite time.

Proof. First Proposition 1.1 gives the existence of unique local solution $\varphi \in C([0, T) ; \tilde{H})$, where $T$ is the existence time of $\varphi$. Let us prove $T<\infty$. Arguing by contradiction, suppose $T=\infty$. Let

$$
F(t)=\int|x|^{2}|\varphi|^{2} \mathrm{~d} x
$$

Then by Proposition 1.2 we have

$$
\begin{aligned}
F^{\prime \prime}(t) & =8 \int\left(|\nabla \varphi|^{2}-|x|^{2}|\varphi|^{2}-\frac{n(p-1)}{2(p+1)}|\varphi|^{p+1}\right) \mathrm{d} x \\
& \leq 8 \int\left(|\nabla \varphi|^{2}-\frac{n(p-1)}{2(p+1)}|\varphi|^{p+1}\right) \mathrm{d} x=\frac{8}{a} I_{a}(\varphi) .
\end{aligned}
$$


In order to finish this proof we consider the following two cases:

(i) $0<E\left(\varphi_{0}\right)<d$.

In this case from Theorem 3.1 we have $\varphi \in V_{\delta}$ for $\delta_{1}<\delta<\delta_{2}$ and $0 \leq t<\infty$, where $\delta_{1}<\delta_{2}$ are two roots of equation $d(\delta)=E\left(\varphi_{0}\right)$. Clearly we have $\delta_{2}>a>1$. Hence we have $I_{\delta}(\varphi)<0$ and $\|\nabla \varphi\|>r(\delta)$ for $a<\delta<\delta_{2}$, $0 \leq t<\infty$. And $I_{\delta_{2}}(\varphi) \leq 0,\|\nabla \varphi\| \geq r\left(\delta_{2}\right)$ for $0 \leq t<\infty$. Thus from (4.3) we get

$$
\begin{aligned}
F^{\prime \prime}(t) & \leq \frac{8}{a} I_{a}(\varphi)=\frac{8}{a}\left(\left(a-\delta_{2}\right)\|\nabla \varphi\|^{2}+I_{\delta_{2}}(\varphi)\right) \\
\leq & \frac{8}{a}\left(a-\delta_{2}\right)\|\nabla \varphi\|^{2} \leq \frac{8}{a}\left(a-\delta_{2}\right) r^{2}\left(\delta_{2}\right)=-C\left(\delta_{2}\right)<0, \\
F^{\prime}(t) & \leq-C\left(\delta_{2}\right) t+F^{\prime}(0), 0 \leq t<\infty,
\end{aligned}
$$

Hence there exists a $t_{0} \geq 0$ such that $F^{\prime}(t)<F^{\prime}\left(t_{0}\right)<0$ for $t_{0}<t<\infty$ and

$$
F(t) \leq F^{\prime}\left(t_{0}\right)\left(t-t_{0}\right)+F\left(t_{0}\right), t_{0} \leq t<\infty .
$$

Since $I\left(\varphi_{0}\right)<0$ implies $F(0)>0$ from (4.4) it follows that there exists a $T_{1}>0$ such that $F(t)>0$ for $0 \leq t<T_{1}$ and

$$
\lim _{t \rightarrow T_{1}} F(t)=0,
$$

which together with

$$
\left\|\varphi_{0}\right\|^{2}=\|\varphi\|^{2} \leq \frac{2}{n}\|\nabla \varphi\| F^{\frac{1}{2}}(t)
$$

gives

$$
\lim _{t \rightarrow T_{1}} \sup \|\nabla \varphi\|=+\infty
$$

This contradicts $T=+\infty$.

(ii) $E\left(\varphi_{0}\right) \leq 0$.

Since $I\left(\varphi_{0}\right)<0$ implies $\left\|\varphi_{0}\right\| \neq 0$, from Theorem 3.3 we have $\varphi \in V_{\delta}$ for $0<\delta<\frac{p+1}{2}, 0 \leq t<\infty$. If in the proof of part (i) $\delta_{2}$ is replaced by $\frac{p+1}{2}$, then we also obtain $T<\infty$.

Finally from Proposition 1.1 we get

$$
\lim _{t \rightarrow T}\|\varphi\|_{H}=+\infty
$$

Theorem 4.6 is proved.

Remark 4.7. The proof of Theorem 4.6 strongly depends on the fact that $\varphi(t) \in V_{\delta}$ for some $\delta>a$, where $\varphi(t)$ is the solution of problem (1.1) with $E\left(\varphi_{0}\right)<d, I\left(\varphi_{0}\right)<0$. Therefore the introducing of $V_{\delta}$ is crucial for the proof of Theorem 4.6.

Corollary 4.8. Let $p$ satisfy (A), $\varphi_{0} \in H$ and $a<\delta<\frac{p+1}{2}$. Assume that $E\left(\varphi_{0}\right) \leq d(\delta)$ and $I_{\delta}\left(\varphi_{0}\right)<0$. Then solution of problem (1.1) blows up in finite time.

Proof. On the one hand, from $E\left(\varphi_{0}\right) \leq d(\delta)$ and Corollary 2.5 we get $E\left(\varphi_{0}\right)<d$. On the other hand, from $I_{\delta}\left(\varphi_{0}\right)<0$ we get $I\left(\varphi_{0}\right)<0$. So by Theorem 4.6 solution of problem (1.1) blows up in finite time.

Corollary 4.9. Let $p$ satisfy (A), $\varphi_{0} \in H$. Assume that $E\left(\varphi_{0}\right)<0$ or $E\left(\varphi_{0}\right)=0, \varphi_{0} \neq 0$. Then solution of problem (1.1) blows up in finite time.

Proof. This corollary follows from Theorem 3.3 and Theorem 4.6.

Next we discuss the blow up of solutions for problem (1.1) with $E\left(\varphi_{0}\right)=d$.

Theorem 4.10. Let $p$ satisfy $(A), \varphi_{0} \in H$. Assume that $E\left(\varphi_{0}\right)=d, I\left(\varphi_{0}\right)<0$ and $F^{\prime}(0) \leq 0$. Then solution of problem (1.1) blows up in finite time. 
Proof. Let $\varphi(t)$ be any solution of problem (1.1) with $E\left(\varphi_{0}\right)=d, I\left(\varphi_{0}\right)<0$ and $F^{\prime}(0) \leq 0$. Let us prove $T<\infty$. Arguing by contradiction, again suppose $T=+\infty$. From Theorem 3.2 we have $\varphi(t) \in V$ for $0 \leq t<\infty$. Hence we get

$$
F^{\prime \prime}(t) \leq \frac{8}{a} I(\varphi)<0,0 \leq t<\infty .
$$

From this and $F^{\prime}(0) \leq 0$ it follows that for any $t_{0}>0$ we have $F^{\prime}\left(t_{0}\right)<0$ and

$$
F(t)<F^{\prime}\left(t_{0}\right)\left(t-t_{0}\right)+F\left(t_{0}\right), t_{0}<t<\infty .
$$

Hence there exists a $T_{1}>0$ such that $F(t)>0$ for $0 \leq t<T_{1}$ and

$$
\lim _{t \rightarrow T_{1}} F(t)=0 .
$$

The remainder of this proof is same as the proof of Theorem 4.6.

From Theorem 4.1 and Theorem 4.6 we can obtain a sharp condition for global existence of solutions for problem (1.1) with $E\left(\varphi_{0}\right)<d$ as follows:

Theorem 4.11. Let $p$ satisfy $(A), \varphi_{0} \in H$. Assume that $E\left(\varphi_{0}\right)<d$. Then when $I\left(\varphi_{0}\right)>0$, solution of problem (1.1) exists globally; and when $I\left(\varphi_{0}\right)<0$, solution of problem (1.1) blows up in finite time.

From Corollary 4.3 and Corollary 4.8 we can obtain a serious of sharp conditions for global existence and finite time blow up of solution for problem (1.1) in the following theorem.

Theorem 4.12. Let $p$ satisfy (A), $\varphi_{0} \in H, a<\delta<\frac{p+1}{2}$. Assume that $E\left(\varphi_{0}\right) \leq d(\delta)$. Then when $I_{\delta}\left(\varphi_{0}\right)>0$, the solution of problem (1.1) exists globally; and when $I_{\delta}\left(\varphi_{0}\right)<0$, solution of problem (1.1) blows up in finite time.

From Theorem 4.1 and Theorem 4.10 we can obtain the following sharp condition for global existence of solution for problem (1.1) with $E\left(\varphi_{0}\right)=d$.

Theorem 4.13. Let $p$ satisfy $(A), \varphi_{0} \in H$. Assume that $E\left(\varphi_{0}\right)=d, F^{\prime}(0) \leq 0$. Then when $I\left(\varphi_{0}\right)>0$, the solution of problem (1.1) exists globally; and when $I\left(\varphi_{0}\right)<0$, solution of problem (1.1) blows up in finite time.

Note that from Lemma 2.4 we have

$$
d=d(a) \geq \frac{n(p-1)-4}{2 n(p-1)} r^{2}(a)=d_{0} .
$$

From this we get the following sharp condition that only depends on $\left\|\nabla \varphi_{0}\right\|$.

Corollary 4.14. Let $p$ satisfy (A), $\varphi_{0} \in H$. Assume that $E\left(\varphi_{0}\right)<d_{0}$. Then when $\left\|\nabla \varphi_{0}\right\|<r(a)$, solution of problem (1.1) exists globally. When $\left\|\nabla \varphi_{0}\right\| \geq r(a)$, solution of problem (1.1) blows up in finite time.

Proof. If $\left\|\nabla \varphi_{0}\right\|<r(a)$, then $0<\left\|\nabla \varphi_{0}\right\|<r(a)$ or $\left\|\nabla \varphi_{0}\right\|=0$, i.e. $I\left(\varphi_{0}\right)>0$ or $\left\|\nabla \varphi_{0}\right\|=0$. Then Theorem 4.1 gives the existence of unique global solution $\varphi(t) \in C([0, \infty) ; \widetilde{H})$. If $\left\|\nabla \varphi_{0}\right\| \geq r(a)$, then by

$$
\begin{aligned}
& \frac{1}{2}\left\||x| \varphi_{0}\right\|^{2}+\frac{n(p-1)-4}{2 n(p-1)}\left\|\nabla \varphi_{0}\right\|^{2}+\frac{1}{p+1} I\left(\varphi_{0}\right) \\
= & E\left(\varphi_{0}\right)<d_{0}=\frac{n(p-1)-4}{2 n(p-1)} r^{2}(a),
\end{aligned}
$$

we get $I\left(\varphi_{0}\right)<0$. Hence from Theorem 4.6 it follows that solution of problem (1.1) blows up in finite time.

Corollary 4.15. Let $p$ satisfy (A), $\varphi_{0} \in H$. Assume that $E\left(\varphi_{0}\right)>0$ and

$$
\frac{n(p-1)}{2(p+1)}\left\|\varphi_{0}\right\|^{p+1-\frac{n(p-1)}{2}}\left(\frac{2 n(p-1)}{n(p-1)-4} E\left(\varphi_{0}\right)\right)^{\frac{n(p-1)-4}{4}}<C_{\star}^{-1} .
$$


Then when

$$
\frac{n(p-1)}{2(p+1)}\left\|\varphi_{0}\right\|^{p+1-\frac{n(p-1)}{2}}\left\|\nabla \varphi_{0}\right\|^{\frac{n(p-1)-4}{2}}<C_{\star}^{-1},
$$

the solution of problem (1.1) exists globally. And when

$$
\frac{n(p-1)}{2(p+1)}\left\|\varphi_{0}\right\|^{p+1-\frac{n(p-1)}{2}}\left\|\nabla \varphi_{0}\right\|^{\frac{n(p-1)-4}{2}} \geq C_{\star}^{-1},
$$

solution of problem (1.1) blows up in finite time.

Proof. This corollary follows from Corollary 4.14 and the fact that (4.5), (4.6) and (4.7) are equivalent to $E\left(\varphi_{0}\right)<$ $d_{0},\left\|\nabla \varphi_{0}\right\|<r(a)$ and $\left\|\nabla \varphi_{0}\right\| \geq r(a)$ respectively.

From $E\left(\varphi_{0}\right)<\frac{1}{2}\left(\left\||x| \varphi_{0}\right\|^{2}+\left\|\nabla \varphi_{0}\right\|^{2}\right)$ for $\varphi_{0} \neq 0$ and Corollary 4.15 we can obtain the following corollary.

Corollary 4.16. Let $p$ satisfy (A), $\varphi_{0} \in H$. Assume that $E\left(\varphi_{0}\right)>0$ and

$$
\frac{n(p-1)}{2(p+1)}\left\|\varphi_{0}\right\|^{p+1-\frac{n(p-1)}{2}}\left(\frac{n(p-1)}{n(p-1)-4}\left(\left\||x| \varphi_{0}\right\|^{2}+\left\|\nabla \varphi_{0}\right\|^{2}\right)\right)^{\frac{n(p-1)-4}{4}} \leq C_{\star}^{-1},
$$

then solution of problem (1.1) exists globally.

Proof. One can see that (4.8) gives both (4.5) and (4.6), which proves this corollary.

Finally we give another series of sharp conditions for globally existence and finite time blow up of solution for problem (1.1) as follows:

Corollary 4.17. Let $p$ satisfy (A), $\varphi_{0} \in H, a<\delta<\frac{p+1}{2}$. Assume that $E\left(\varphi_{0}\right) \leq a(\delta) r^{2}(\delta)$, where $r(\delta)$ and $a(\delta)$ are defined in Lemma 2.2 and Lemma 2.4. Then when $\left\|\nabla \varphi_{0}\right\|<r(\delta)$ the solution of problem (1.1) exists globally; and when $\left\|\nabla \varphi_{0}\right\| \geq r(\delta)$ the solution of problem (1.1) blows up in finite time.

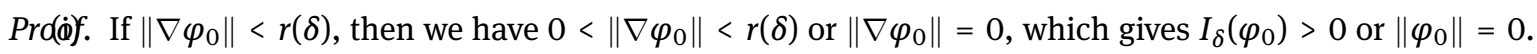
Hence by Theorem 4.12 and Theorem 4.1 the solution of problem (1.1) exists globally.

(ii) If $\left\|\nabla \varphi_{0}\right\| \geq r(\delta)$, then from $\left\||x| \varphi_{0}\right\|>0$ and

$$
\frac{1}{2}\left\||x| \varphi_{0}\right\|^{2}+a(\delta)\left\|\nabla \varphi_{0}\right\|^{2}+\frac{1}{p+1} I_{\delta}\left(\varphi_{0}\right)=E\left(\varphi_{0}\right) \leq a(\delta) r^{2}(\delta),
$$

we get $I_{\delta}\left(\varphi_{0}\right)<0$. Again by Theorem 4.12 solution of problem (1.1) blows up in finite time.

Acknowledgement: We appreciate the reviewers for their precious comments and suggestions, which help us tremendously to improve our manuscript.

\section{References}

[1] C.C. Bradley, C.A. Sackett, R.G. Hulet, Bose-Einstein condensation of lithium: Observation of limited condensate number, Phys. Rev. Lett., 78 (1997), 985-989.

[2] R. Carles, Remarks on the nonlinear Schrödinger equation with harmonic potential, Ann. H. Poincaré,3 (2002), no. 4, 757772.

[3] R. Carles, Critical nonlinear Schrödinger equations with and without harmonic potential, Math. Models Methods Appl. Sci., 12 (2002), 1513-1523.

[4] T. Cazenave, An Introduction to Nonlinear Schrödinger Equations, Textos de Metodos Matematicos, Rio de Janeiro, 26 (1996).

[5] G. Chen, J. Zhang, Remarks on global existence for the supercritical nonlinear Schrödinger equation with a harmonic potential, J. Math. Anal. Appl., 320 (2006), no. 2, 591-598. 
[6] F. Dalfovo, S. Giorgini, Pitaevskii, P. Lev, et al., Theory of Bose-Einstein condensation in trapped gases, Rev. Modern Phys., 71 (1999), no. 3, 463-512.

[7] D. Fujiwara, Remarks on convergence of the Feynman path integrals, Duke Math. J., 47 (1980), no. 3, 559-600.

[8] J. Ginibre, G. Velo, On a class of nonlinear Schrödinger equations, J. Funct. Anal., 32 (1979), no. 1, 33-71.

[9] J. Ginibre, G. Velo, The global Cauchy problem for the nonlinear Schrödinger equation, revisited, Ann. Inst. H. Poincaré Anal. Non Linéaire, 2 (1985), no. 4, 309-327.

[10] Y. Kagan, A.E. Muryshev, G.V. Shlyapnikov, Collapse and Bose-Einstein condensation in a trapped Bose gas with negative scattering length, Phys. Rev. Lett., 81 (1998), 933-937.

[11] J.L. Lebowitz, H.A. Rose, E.R. Speer, Statistical mechanics of the nonlinear Schrödinger equation, J. Statist. Phys., 54 (1989), no. 1, 657-687.

[12] Y. Liu, On potential wells and vacuum isolating of solutions for semilinear wave equations,Journal of Differential Equations, 192 (2003), no. 1, 155-169.

[13] Y. Liu, J. Zhao, On potential wells and applications to semilinear hyperbolic equations and parabolic equations, Nonlinear Analysis, 64 (2006), no. 12, 2665-2687.

[14] Y. Liu, R. Xu, Wave equations and reaction-diffusion equations with several nonlinear source terms of different sign, Discrete and Continuous Dynamical System-Series B, 7 (2007), 171-189.

[15] Y. Liu, R. Xu, Remarks on nonlinear Schrödinger equation with harmonic potential, Journal of Mathematical Physics, 49 (2008), 043512.

[16] Y. G. Oh, Cauchy problem and Ehrenfes't law of nonlinear Schrödinger equations with potentials, J. Differential Equations, 81 (1989), 255-274.

[17] J. Shu, J. Zhang, Nonlinear Schrödinger equation with harmonic potential, Journal of Mathematical Physics, 47(2006), 063503.

[18] T. Tsurumi, M. Wadati, Collapses of wave functions in multidimensional nonlinear Schrödinger equations under harmonic potential, Phys. Soc. Jpn., 66 (1997), 3031-3034.

[19] M.I. Weinstein, Nonlinear Schrödinger equations and sharp interpolations estimates, Comm. Math. Phys., 87 (1983), $567-$ 576.

[20] K. Yajima, On fundamental solution of time dependent Schröinger equations, Contemp. Math., 217 (1998), 49-68.

[21] J. Zhang, Stability of attractive Bose-Einstein condensates, J. Statist. Phys., 101 (2000), 731-746.

[22] J. Zhang, Stability of standing waves for nonlinear Schrödinger equations with unbounded potentials, Z. Angew. Math. Phys., 51 (2000), 498-503.

[23] J. Zhang, Sharp threshold for blowup and global existence in nonlinear Schrödinger equations under a harmonic potential, Comm. Partial Differential Equations, 30 (2005), 1429-1443.

[24] Z. Gan, J. Zhang, Sharp threshold of global existence and instability of standing wave for a Davey-Stewartson system. Comm. Math. Phys., 283 (2008), 93-125.

[25] Carles R., Markowich P.A., C. Sparber, On the Gross-Pitaevskii equation for trapped dipolar quantum gases. Nonlinearity, 21 (2008), 2569-2590.

[26] S. Le Coz, R. Fukuizumi, G. Fibich, B. Ksherim, Y. Sivan, Instability of bound states of a nonlinear Schrödinger equation with a Dirac potential. Phys. D, 237 (2008), 1103-1128.

[27] X. Song, Sharp thresholds of global existence and blowup for a system of Schrödinger equations with combined powertype nonlinearities. J. Math. Phys., 51 (2010), 033509.

[28] Z. Gan, J. Zhang, Nonlocal nonlinear Schrödinger equations in $\mathbb{R}^{3}$. Arch. Ration. Mech. Anal., 209 (2013), 1-39.

[29] J. Huang, J. Zhang, X. Li, Stability of standing waves for the $L^{2}$-critical Hartree equations with harmonic potential. Appl. Anal., 92 (2013), 2076-2083.

[30] A. Poiret, R. Didier, T. Laurent, Probabilistic global well-posedness for the supercritical nonlinear harmonic oscillator. Anal. PDE, 7 (2014), 997-1026.

[31] Z. Gan, Standing wave and global existence to nonlocal nonlinear Schr'odinger equations: the two-dimensional case. Nonlinearity, 29 (2016), 2050-2081.

[32] J. Huang, J. Zhang, Exact value of cross-constrain problem and strong instability of standing waves in trapped dipolar quantum gases. Appl. Math. Lett., 70 (2017), 32-38. 